\title{
Three-Dimensional FDTD Model to Study the Elastic-Wave Interaction with Buried Land Mines
}

\author{
Christoph T. Schröder ${ }^{(a)}$ and Waymond R. Scott, Jr. ${ }^{(b)}$ \\ School of Electrical and Computer Engineering \\ Georgia Institute of Technology \\ Atlanta, GA 30332-0250, USA \\ (a) christoph.schroeder@ee.gatech.edu, 404-894-3123
}

(b) waymond.scott@ee.gatech.edu, 404-894-3048

\begin{abstract}
A three-dimensional finite-difference model for elastic waves in the ground has been developed and implemented. The model is used to investigate the interaction of elastic waves with buried land mines. When elastic waves interact with a buried mine, a strong resonance occurs at the mine location. The resonance can be used to enhance the mine's signature and to distinguish the mine from clutter. Results are presented for a single mine buried in the ground. The predictions of the numerical model are in fairly good agreement with experimental results.
\end{abstract}

\section{INTRODUCTION}

A new technique is being investigated at the Georgia Institute of Technology, in which buried land mines are located by using both elastic (acoustic) and electromagnetic waves in a synergistic manner [1]-[4]. Here, elastic waves interact with a buried land mine and cause the mine and the surface above the mine to vibrate. An electromagnetic radar records the vibrations and, thus, detects the mine. During the process of developing the elastic/electromagnetic sensor, a major part has been the implementation of a numerical model which simulates the interaction of the elastic waves with the buried land mines.

The numerical model is based on the finite-difference time-domain (FDTD) method. The equation of motion and the stress-strain relation, together with a constitutive relation, form a set of first-order partial differential equations that completely describes the elastic wave motion in a medium. Introducing finite differences, this set of equations can be discretized and adapted to the finite-difference time-domain modeling scheme.

The finite-difference model has been implemented in two and three dimensions. The numerical model is used to investigate the mine-wave interactions and has been very helpful for explaining and understanding the experimental results. In this paper, results obtained with the threedimensional model are presented. The interaction of elastic waves with a buried antipersonnel mine is shown.

\section{THREE-DIMENSIONAL NUMERICAL MODEL}

In a realistic setting, one or several mines are buried in the ground, surrounded and covered by various kinds of clutter. To approximate these conditions, experiments have been performed with mines buried in a large sand box [1], [2]. In these experiments, elastic waves are launched by an electrodynamic transducer placed on the surface of the ground. The waves propagate along the surface and interact with the buried land mines. To study these mine-wave interactions, a three-dimensional finitedifference model has been developed.

\section{Finite-Difference Model}

Fig. 1 shows the three-dimensional finite-difference model. To reasonably simplify the model, the ground is assumed to be linear, isotropic and lossless. The surface of the ground is modeled as a free-surface, and a Perfectly Matched Layer terminates the solution space at the remaining grid edges and absorbs the outward traveling waves [5]. The solution space is discretized using a staggered finite-difference grid.

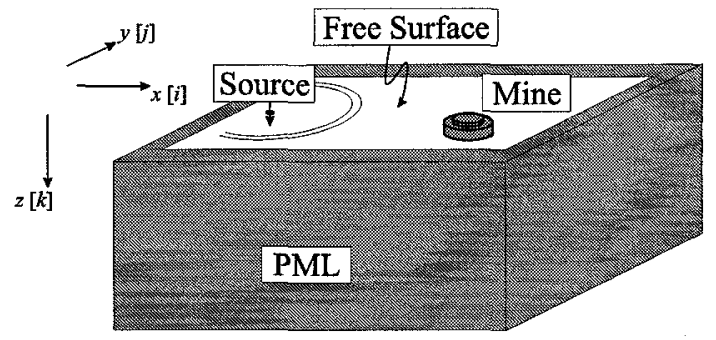

(a)

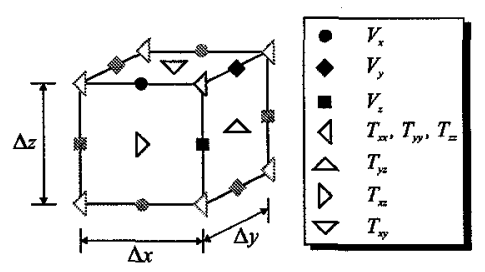

(b)

Fig. 1. Three-dimensional finite-difference model; (a) layout, (b) finite-difference cell.

The elastic wave motion in solids is described by a set of fundamental partial-differential equations: the equation of motion relating the particle velocity vector and the mechanical stress tensor, the strain-velocity relation and the elastic constitutive relation. Combining these equations, a first-order system of equations is obtained describing the elastic wave fields entirely in terms of the particle velocity and the mechanical stress. In three dimensions, nine 
equations for three unknown velocity components and six unknown stress tensor components arise.

The equations are discretized by introducing finitedifferences. The discretization leads to the characteristic finite-difference grid. In this grid, the field components are staggered in space and time. The finite-difference grid can be thought of as being comprised of basis cells. The three-dimensional basis cell for the elastodynamic case resembles strongly the three-dimensional basis cell for electromagnetic finite-difference modeling, the so-called Yeecell. However, due to the stress being a tensor, more field components are present in the elastodynamic case. Fig. 1 (b) shows the three-dimensional finite-difference basis cell. Note that the field components are not known at the same points in space and time. The grid is laid out such that each field component is surrounded by the field components it is dependent on.

The finite-difference model has been implemented in a fully parallel fashion. The computations for this paper's results have been performed both on a Cray T3E parallel supercomputer located at the ERDC Massively Shared Resource Center in Vicksburg, Mississippi, and on a Beowulf PC cluster located at the Georgia Institute of Technology. The Beowulf cluster has been developed and built especially for the model described in this paper.

\section{INTERACTION OF ELASTIC WAVES WITH BURIED ANTIPERSONNEL MINES}

The interaction of elastic waves with antipersonnel mines, buried in sand, is to be investigated. In the experiment, the mines are buried in a large sand-filled box. If the sand is assumed to be linear, isotropic and lossless, its properties can be described by three parameters: the material density, $\rho=1400 \mathrm{~kg} / \mathrm{m}^{3}$, the pressure wave speed, $c_{p}=250 \mathrm{~m} / \mathrm{s}$, and the shear wave speed. The shear wave speed is not constant and increases with depth.

The space step for the numerical model is chosen to be $\Delta x=\Delta y=\Delta z=0.5 \mathrm{~cm}$; the time step is $\Delta t=11.54 \mu \mathrm{s}$ and, thus, fulfills the Courant condition (the necessary condition for stability of the finite-difference algorithm). The excitation has the shape of a Gaussian pulse. To be able to compare the numerical and experimental results, the transfer function of the system is determined in a postprocessing step for each point in space and convolved with the transducer motion as measured in the experiment.

To investigate the interactions with a buried land mine, a simple model for an antipersonnel mine is inserted into the numerical model. Fig. 2 shows the simple model, together with a simplified cross-sectional drawing of a real TS-50 antipersonnel mine. The simple mine model consists of a main chamber containing plastic explosives, and a smaller chamber on top of the mine's main chamber filled with air. The air-filled chamber is inserted into the model to coarsely approximate the structure of a real TS-50 mine (see Fig. 2 (a)).

Fig. 3 shows waterfall graphs of the mine-wave interaction for both experiment and numerical simulation. In these graphs, the vertical particle displacement at a num-

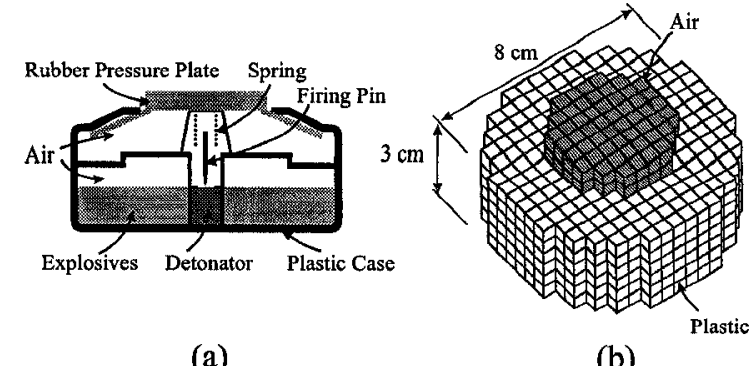

(a)

Fig. 2. (a) Cross-sectional drawing of a real TS-50 mine; (b) simple model.

ber of points along a line on the surface is plotted as a function of time and offset by the distance from the source. The slope of the traveling waves in the graph indicates the wave speed. Thus, by determining the slope, the different wave types can be distinguished. The mine is buried 2 $\mathrm{cm}$ beneath the surface, at a distance of $70 \mathrm{~cm}$ from the source. A pressure wave $(\mathrm{P})$ and a Rayleigh surface wave (R) arise. The waves hit the mine and are reflected (rR) and transmitted. While the interaction of the mine with the pressure wave is weak, the surface wave strongly interacts with the mine. In both experiment and numerical simulation, resonant oscillations occur at the mine location and remain even after the waves have passed the mine. For the numerical model, it can be shown that the incident waves couple into flexural waves which arise in the thin soil layer above the mine [4]. These flexural waves are confined to the thin layer and form a standing wave pattern, giving rise to the resonant oscillations. While this explains the resonance in the numerical model, it gives only one possible cause for the resonance in the experiment. A real TS-50 mine has several chambers, it has a flexible case that can support both flexural and longitudinal waves, and it contains springs that can also give rise to resonances. The authors are currently working on refining the numerical model to incorporate more details of the mine.

In Fig. 4, the vertical particle displacement on the surface and on a cross section through the ground is shown in some pseudo-color plots as obtained with the numerical model. The upper plots show the wave fields on the surface, the lower plots correspond to a cross section through the ground. The surface plane has dimensions of $120 \mathrm{~cm}$ by $80 \mathrm{~cm}$ and the cross section has a size of $120 \mathrm{~cm}$ by 30 $\mathrm{cm}$. The source is located on the surface, off the left edge of the plots. The dynamic range of the plots is $60 \mathrm{~dB}$. The wave fields are shown at four different instances in time, corresponding to the vertical lines indicated by $T_{1}, T_{2}, T_{3}$ and $\mathrm{T}_{4}$ in Fig. 3 (b). In the first plot, the surface wave is seen to just hit the mine. While only the surface wave (R) is visible on the surface, both the surface wave (R) and the shear wave (S) appear on the cross section. In the second plot, the surface wave has just passed the mine. The interaction of the surface wave with the mine gives rise to reflected surface waves ( $\mathrm{rR}$ ) and reflected shear waves (rS), which are clearly visible on the cross section. Pressure waves are also induced by the surface wave, but they 


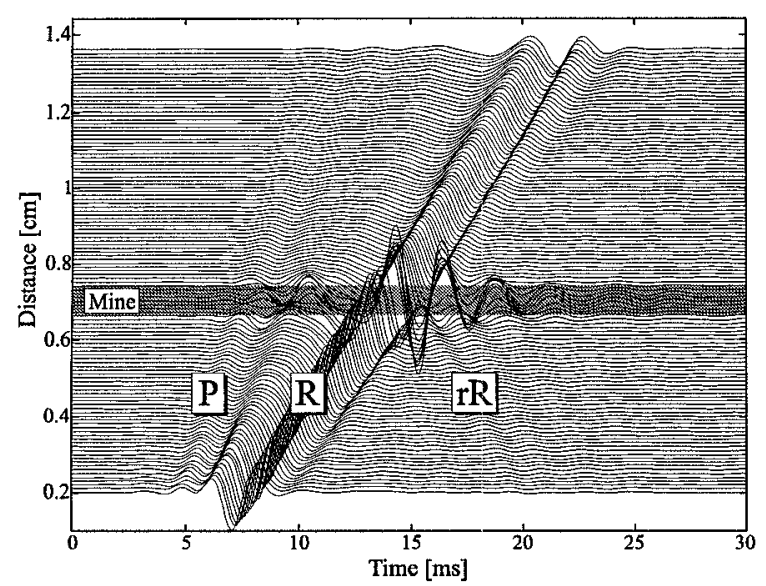

(a)

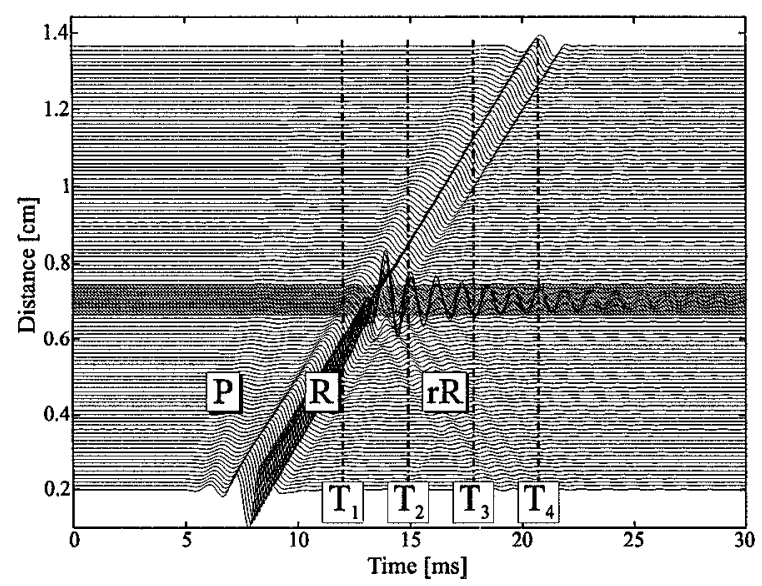

(b)

Fig. 3. Interaction of elastic waves with a buried antipersonnel mine; waterfall graphs of the vertical particle displacement on the surface according to (a) experiment and (b) numerical simulation.

are weak and not visible. In the third and fourth plot, the surface wave has passed the mine. Some energy, however, remains at the mine and causes the mine to vibrate and to radiate.

\section{CONCLUSIONS}

A three-dimensional finite-difference model for elastic waves in the ground has been developed and implemented in a fully parallel fashion. Results are obtained and compared to experimental results. When an antipersonnel mine interacts with elastic waves, a strong resonance occurs at the mine location. This resonance has been observed in both experiment and numerical simulation.

\section{REFERENCES}

[1] W. R. Scott, Jr., C. T. Schröder, and J. S. Martin, "An acousto-electromagnetic sensor for locating land mines," in Detection and Remediation Technologies

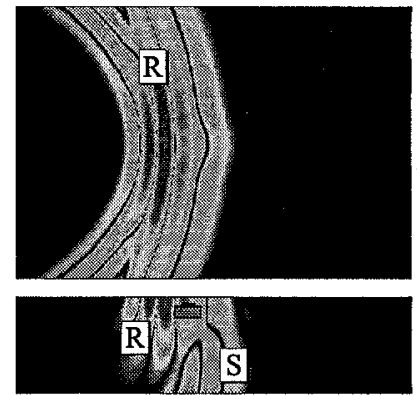

$\mathrm{T}_{1}$

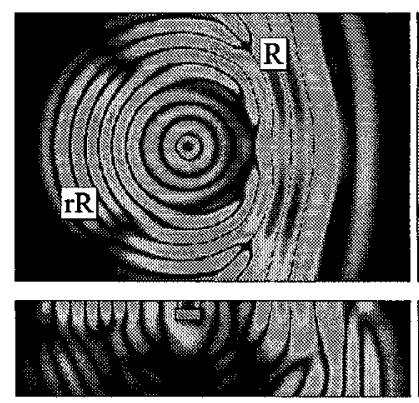

$\mathrm{T}_{3}$
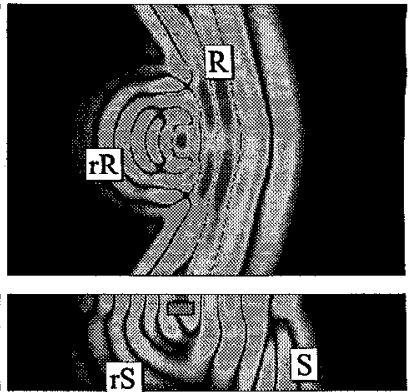

$\mathrm{T}_{2}$

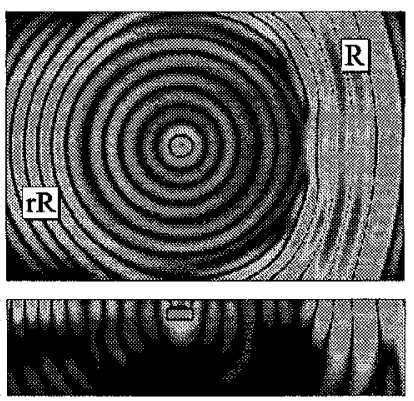

$\mathrm{T}_{4}$
Fig. 4. Interaction of elastic waves with a buried antipersonnel mine; pseudo color plots of the normal particle displacement on the surface (top) and on a cross section through the ground (bottom) at four instances in time, corresponding to the vertical lines in Fig. 3 (b).

for Mines and Minelike Targets III, Proc. SPIE, 1998, vol. 3392 , pp. $176-186$.

[2] W. R. Scott, Jr. and J. S. Martin, "Experimental investigation of the acousto-electromagnetic sensor for locating land mines," in Detection and Remediation Technologies for Mines and Minelike Targets IV, Proc. SPIE, 1999.

[3] C. T. Schröder and W. R. Scott, Jr., "Threedimensional FDTD model to study the elastic-wave interaction with buried land mines," in Detection and Remediation Technologies for Mines and Minelike Targets V, Proc. SPIE, 2000.

[4] C. T. Schröder and W. R. Scott, Jr., "A finitedifference model to study the elastic-wave interactions with buried land mines," IEEE Trans. on Geophysics and Remote Sensing, to be published.

[5] W. C. Chew and Q. H. Liu, "Perfectly matched layer for elastodynamics; a new absorbing boundary condition," J. Comput. Acoustics, vol. 4, pp. 341-359, 1996. 